\title{
Ungarische Ophthalmologische Gesellschaft in Budapest
}

Gemeinsame Sitzung mit der Gesellschaft der Spitalärzte am 9. März 192J. Bericht vom Sekretär Dr. G. Horay, Budapest.

Vorsitzende: Prof. Dr. /. v. Imre sen. und Prof. Dr. K. v. Borszéky. Vorstellungen:

1. /. v. Csapody: a) Skleraresektion wegen Netzhautablösung. Hat im vorgestellten Fall die

Müllersche Operation sechs Wochen nach dem Entstehen der Netzhautablösung ausgeführt. Vorher zweimal Sklera-punktion mit vorübergehendem Erfolge. Nach der Resektion hat die Netzhaut sich teilweise zurückgelegt, die Sehschärfe erreichte 5/50. An-scheinend wirkte die Operation nicht durch die Verminderung der zwi-schen Sklera und Augeninhalt bestehenden räumlichen Mißverhältnisse, sondern dadurch, daß die Retina an der Stelle der Operation fixiert wurde.

Ungarische Ophthalmologische Gesellschaft in Budapest. 4H

b) Fall von multiplem Epitheliom. Je ein Geschwür auf beiden Unterlidern, an der rechten Schläfe, am Nasenrücken und auf der oberen Lippe. In der Gesichtshaut mehrere auf Gummata verdächtige Narben. WaR. positiv. Die histologische Untersuchung erwies keinen Zusammenhang mit den luetischen Narben. Das ausgeheilte Trachom kann viel-leicht für das Entstehen der Geschwüre am unteren Lide den Grund vorbereitet haben. Pat. wurde seit drei Wochen mit Radium bestrahlt, seither starke Rückbildung der Neubildungen. Über die weitere Therapie kann erst nach Ablauf der Reaktion entschieden werden.

2. L. v. Liebermann: a) Zwei Fälle von höchstgradigem Konvergenz-schielen (50 bzw. $45^{\circ}$ ) durch Vorlagerung nur des einen äußeren geraden Muskels und ohne Eingriff am Schielmuskel vollkommen berichtigt, mittels gedoppelter Ziegler-Mellerscher Naht. (Kurz referiert im Ztrbl. f. d. ges. Ophth. 14, 332, wird demnächst ausführlich mitgeteilt.)

Vier Fälle von Fetteinpflanzung nach Enukleation, um zu zeigen, daß bei richtiger Ausführung Resorption des Fettes nicht zu befürchten ist. (Die Pat. wurden vor ein bis drei Jahren operiert.) Sitz der Prothesen tadellos. Kompliziertere Verfahren, wie Einpflanzung von Knochen, Knorpel oder fremdem Material haben demnach kerne Berechtigung.

Nacnstardiszission mit schmalem Graeïemesser. Dieses wiederholt von verschiedenen Autoren vorgeschlagene Verfahren verdient berück-sichtigt zu werden. Noch besser sind ganz schmale zweischneidige Messer, wie sie Elschnig vor Jahren angewendet und wieder verlassen hat. Man bekommt solche Messer leichter tadellos scharf, auch die längere Schneide und die bessere Anpassung an den Wundkanal als bei den Diszissions-messern ist ein Vorteil. Man operiert ohne Abfluß des Kammerwassers (mit Diszissionsmesser nicht immer)..

Trachom und kongenital-luetische Keratitis gleichzeitig rasch geheilt. Das Trachom (hochgradiges Körnertrachom) wurde mittels Ausquetschung und täglicher Massage mit jenem neuen Präparat be-handelt, über welches Vortr. in der Sitzung der U.O.G. am 28. Jan. ds. Js. berichtete; die Keratitis parenchymatosa mit kombinierter Milch-Hg-Neosalvarsan-Behandlung. Dieses Verfahren wirkt mit wenigen Aus-nahmen prompt; beginnende Fälle heilen oft abortiv. Der in fast alien Lehrbüchern immer noch vertretene Standpunkt, die Keratitis parenchymatosa sei eine fast jeder Therapie trotzende Erkrankung, besteht längst nicht mehr zu Recht, wie dies 
Vortr. schon öfters betont hat. - Kombination von Ker. parench. mit Trachom ist nicht häufig, die Ansicht einiger Autoren, die in der Lues congenita ein besonders disponierendes Moment für Trachomerkrankung erblicken, ist also falsch.

Vortr age: 1. E. v. Grósz: Die Vererbung in der Augenheilkunde. Befaßt sich erst mit allgemeinen Regeln der Vererbungslehre und betont die Schwie-rigkeiten. Erwähnt die bekannte Hemeralopie-Familie Nougaret. Dann folgen Betrachtungen über die Vererbung der Farbenblindheit, der De-generatio pigm. retinae, des Glaukoms, der Myopie. Zeigt einige Stamm-bäume, u. a. den Stammbaum einer Familie, wo der Star durch vier Generationen sich vererbte. Betont die Wichtigkeit der Vererbungslehre in der allgemeinen Pathologie. Ungarische Ophthalmologische Gesellschaft in Budapest.

2. A. v. Sarbó: Neuere ophthalmologische Beziehungen in der Neurologie. Auf Grund von Erfahrungen der Enzephalitis haben wir einen neuen Typus der Bewegungsstörungen kennen gelernt: die extrapyra-midalen Bewegungsstörungen. Das Auge gibt zum Studium dieser Störungen gute Gelegenheit. Vortr. bespricht der Reihe nach die Bewegungsstörungen des Auges: das Lidflackern, das Fehlen der Konvergenz, den , haftenden Blick", den Tic. Alle diese Störungen werden vom Vortr. mit der gestörten Funktion des Striatum erklärt. Zu diesen Funktionen gehört auch das statische Gleichgewicht des Körpers, dessen Zentrum der rote Kern ist. Faßt das Rombergsche Phänomen als eine Funktions-störung der roten Kerne auf und empfiehlt um ein wichtiges diagnostisches Frühzeichen zu erhalten, der gewohnten regelmäßigen Untersuchung das Zurückdrücken des Kopfes anzuschließen. Dadurch ermöglicht man in Fallen von Tabes oder Sklerosis multiplex schon frühzeitig die Diagnose einer statischen Gleichgewichtsstörung.

L. v. Liebermann zeigt verschiedene praktische Beleuchtungsein-richtungen zur Untersuchung, Demonstration und zur Operationsbe-leuchtung. (Zum Referat nicht geeignet; einer ausführlichen Mitteüung vorbehalten.)

A. Licskô: Behandlung von Augenerkrankungen mit Eigenblut-injektionen. Vortr. befaßt sich erst mit den verschiedenen Anwendungs-formen des Eigenblutes und bespricht die Stellung, die diese Behandlung in der Reiztherapie einnimmt. In der Augenklinik Nr. I wird Eigenblut seit zwei Jahren intragluteal und subkonjunktival eingespritzt. Die darauffolgende Reaktion besteht aus einer allgemeinen und aus einer lokalen vorübergehenden Leukozytose. Das Eigenblut hat sich am besten gegen Iritis und Iridozyklitis verschiedener Ätiologie, weiter gegen Chorioiditis und akute hämorrhagische Iritis bewährt. Rechtzeitig ge-geben, ist es auch bei infektiösen Verletzungen von Nutzen. Sehr gute Erfolge bekommt man, wenn 1 - 2 Milchinjektionen 8-10 Eigenblut-injektionen angeschlossen werden.

Diagnose und Therapie.

M. M. Cruickshank, Major. Beobachtungen über die Komplikationen bei 4000 Staroperationen. British Journal of Ophthalmology Bd. 11, 1927.

S.275.

4. 4000 Staroperationen wurden in Shikarpur (Indien) von Dr. Holland und einem Stabe von augenärztlichen Mitarbeitern

darunter dem Ver-fasser

in den Operationszeiten der Jahre 1923/24 und 1926 (je 7 Wochen im Januar und Februar)

vollführt. Es war Vorsorge getroffen

daß die klinischen Besonderheiten der Fälle und alle

auch die geringsten Ereignisse während und nach der Operation in ein Kataraktbuch ein-getragen und Auszüge hiervon in Form von Krankenkarten hergestellt wurden. Die Kranken strömten von 
weither zusammen und kehrten bald in feme Gegenden wieder zurück. Dem Verf. fiel die Aufgabe zu, 